\title{
DISTRIBUTION OF INTEGER LATTICE POINTS IN A BALL CENTRED AT A DIOPHANTINE POINT
}

\author{
HYUNSUK KANG AND ALEXANDER V. SOBOLEV
}

Abstract. We study the variance of the fluctuations in the number of lattice points in a ball and in a thin spherical shell of large radius centred at a Diophantine point.

$\S 1$. Introduction. The distribution of lattice points has been extensively studied in the literature for its own sake, as well as with the aim of understanding the clustering of eigenvalues of quantum Hamiltonians associated with integrable systems. The eigenvalues of the "shifted" Laplacian $(-i \nabla-\boldsymbol{\alpha})^{2}, \boldsymbol{\alpha} \in \mathbb{R}^{d}$, $d \geq 2$, on the torus $\mathbb{R}^{d} /(2 \pi \mathbb{Z})^{d}$ are given by the numbers $|\mathbf{m}-\boldsymbol{\alpha}|^{2}, \mathbf{m} \in \mathbb{Z}^{d}$, and hence their counting function coincides with the number $N(t)$ of lattice points inside a ball of a radius $t$, centred at $\boldsymbol{\alpha}$. It is immediately seen that

$$
N(t)=B_{d} t^{d}(1+o(1)), \quad t \rightarrow \infty,
$$

where $B_{d}$ is the volume of the unit ball in $\mathbb{R}^{d}$. Our object is the distribution of $N(t)$, as a function of large $t$ for a fixed $\boldsymbol{\alpha}$, in two regimes. First, we study

$$
F(t):=\frac{N(t)-B_{d} t^{d}}{t^{(d-1) / 2}}
$$

i.e. the normalized deviation of $N(t)$ from its asymptotic value. Secondly, for $\rho \in(0,1)$, we investigate

$$
S(t, \rho):=\frac{N(t+\rho)-N(t)-B_{d}\left((t+\rho)^{d}-t^{d}\right)}{\sqrt{\rho} t^{(d-1) / 2}},
$$

which is the normalized deviation of the number of lattice points in the spherical shell between the spheres of radii $t+\rho$ and $t$ from its asymptotics. Our aim is to study the asymptotics of weighted averages of $F$ and $S$ as $t \rightarrow \infty$ and, in the case of $S$, as $\rho \rightarrow 0$.

Introduce a non-negative function $\omega \in \mathrm{C}_{0}^{\infty}(\mathbb{R})$ such that $\omega(t)=0$ for all $t \leq t_{0}$ with some $t_{0}>0$ and $\int \omega(t) d t=1$. With the smooth measure induced by $\omega$, we define for all $T>0$ an averaging operator for a function $f \in \mathrm{L}_{\text {loc }}^{1}(\mathbb{R})$ by

$$
\langle f\rangle_{T}=\int_{-\infty}^{\infty} f(t) \omega_{T}(t) d t, \quad \omega_{T}(t)=\frac{1}{T} \omega\left(\frac{t}{T}\right) .
$$


If necessary, the dependence on the weight $\omega$ is reflected in the notation: $\langle f\rangle_{\omega, T}$. Regarding $\omega_{T}$ as a distribution density of the "random variable" $t$, we first prove that the expectation values $\langle F\rangle_{T}\left(\langle S(\cdot, \rho)\rangle_{T}\right)$ tend to zero as $T \rightarrow \infty$ $(T \rightarrow \infty, \rho \rightarrow 0)$, and then find the asymptotics of the variances $\left\langle|F|^{2}\right\rangle_{T}$ and $\left\langle|S(\cdot, \rho)|^{2}\right\rangle_{T}$ under appropriate conditions on $T$ and $\rho$.

Throughout the paper we write $a \ll b(a \gg b)$ for two quantities $a$ and $b$ if $a \leq C b(b \leq C a)$ with a positive constant $C$ independent of $t \geq 1, T \geq 1, \rho \geq 0$ and the "smoothing" parameter $M \geq 1$, which will be defined in $\S 2$.

The asymptotic result for the expectation values is relatively simple.

THEOREM 1.1. Let $d \geq 2$. For any $\boldsymbol{\alpha} \in \mathbb{R}^{d}$ we have:

(1) $\langle F\rangle_{T} \rightarrow 0$, as $T \rightarrow \infty$;

(2) if $T^{-Z}<\rho \ll 1$ with some $Z>0$, then $\langle S(\cdot, \rho)\rangle_{T} \rightarrow 0$, as $T \rightarrow \infty$.

The results for the variances require that $\alpha$ should be Diophantine in the following sense.

Definition 1.2. A vector $\alpha \in \mathbb{R}^{d}$ is called Diophantine of type $\kappa$, if there exists a constant $C>0$ such that

$$
\left|\boldsymbol{\alpha}-\frac{\mathbf{m}}{q}\right|>\frac{C}{q^{\kappa}}
$$

for all $\mathbf{m} \in \mathbb{Z}^{d}$ and $q \in \mathbb{N}$.

The smallest possible value of $\kappa$ is $1+d^{-1}$, in which case $\alpha$ is called badly approximable; see [13, pp. 217, 218].

For any non-negative integer $p$, denote

$$
r_{\boldsymbol{\alpha}}(p)=\sum_{\substack{\mathbf{m} \in \mathbb{Z}^{d},|\mathbf{m}|^{2}=p}} \mathrm{e}^{-2 \pi i \boldsymbol{\alpha} \cdot \mathbf{m}} .
$$

The results for the variances are contained in the next two theorems.

THEOREM 1.3. Let $d \geq 2$. Suppose that the components of $(\boldsymbol{\alpha}, 1)$ are linearly independent over $\mathbb{Q}$, and that $\alpha$ is Diophantine of type $\kappa<(d-1)(d-2)^{-1}$. Then the series

$$
\mathfrak{A}_{\boldsymbol{\alpha}}=\sum_{p=1}^{\infty}\left|r_{\boldsymbol{\alpha}}(p)\right|^{2} p^{-(d+1) / 2}
$$

converges and, moreover,

$$
\left\langle|F|^{2}\right\rangle_{T} \rightarrow \frac{1}{2 \pi^{2}} \mathfrak{A}_{\alpha}, \quad T \rightarrow \infty .
$$

For the function $S(t, \rho)$ the formula is more explicit.

THEOREM 1.4. Let $d \geq 2$. Suppose that the components of $(\boldsymbol{\alpha}, 1)$ are linearly independent over $\mathbb{Q}$, and that $\alpha$ is Diophantine of type $\kappa<(d-1)(d-2)^{-1}$. Assume that $\rho \gg T^{-\sigma}$ with some $\sigma \in(0,1)$. Then

$$
\left\langle|S(\cdot, \rho)|^{2}\right\rangle_{T} \rightarrow d B_{d},
$$

as $\rho \rightarrow 0$. 
Note that we regard the formula (6) as asymptotic in two parameters: small $\rho$ and large $T$, related only via the inequality $\rho \gg T^{-\sigma}$. We do not need to assume that $\rho=\rho(T)$ or $T=T(\rho)$.

Observe also that the asymptotic coefficients in (5) and (6) do not depend on the weight $\omega$. This allows one to study the variances of $F(t)$ and $S(t, \rho)$ for the "uniform" distribution density, i.e. when $\omega$ is a characteristic function of an interval, as in $[\mathbf{3 , 5}$. For this it suffices to approximate the characteristic function by smooth weights $\omega$ from below and from above, and use Theorems 1.3 and 1.4.

For $d=2$, the existence of the limiting distribution (as $T \rightarrow \infty$ ) of $F$ was proved for $\boldsymbol{\alpha}=0$ in [6], and this was later generalized to arbitrary $\boldsymbol{\alpha}$ in [2] where the asymptotic formula (5) for $d=2$ was derived as a by-product of the proof; see [2, p. 442]. The case $d \geq 3$ was addressed in [1] where the existence of the limiting distribution of $F$ was announced under the assumption that the vector $\boldsymbol{\alpha}$ is multiplicatively Diophantine, which is a stronger restriction than the Diophantine property in Definition 1.2.

In dimensions $d \geq 3$, the variance of $F$ was studied for $\alpha=0$; see [8] for $d \geq 3$ and also [3] for $d=3$. For $d=3$ and $\alpha=0$, instead of (5), one gets a formula with an extra factor $\log T$ on the right-hand side.

The variance of $S$ has been studied well for $d=2$. A variety of asymptotic formulae were obtained in [5] under the same conditions on the parameters $\rho$ and $T$ as in Theorem 1.4, with various assumptions on the lattice and the vector $\boldsymbol{\alpha}$. In particular, (6) was proved for all Diophantine $\boldsymbol{\alpha}$. For $\boldsymbol{\alpha}=0$, more detailed information about the distribution of $S$ was obtained in [7]: under the condition that $\rho \rightarrow 0$ and $\rho \gg T^{-\delta}$ for all $\delta>0$, it was shown that $S$ has a Gaussian distribution. A similar result was established in [14] for "strongly" Diophantine rectangular lattices.

In the case $\rho t^{d-1} \sim 1$, which is not covered by Theorem 1.4, one recovers (6) from the results of $[\mathbf{1 0}, \mathbf{1 1}]$ under the same conditions on $\boldsymbol{\alpha}$ as in Theorem 1.4. This case is especially interesting since it is related to the so-called BerryTabor conjecture about the distribution of eigenvalues of quantum Hamiltonians associated with integrable systems. We refer to [9-11] for references and discussion.

Our argument follows the usual scheme: first we introduce a "smoothedout" version $F_{M}$ of the quantity $F$, depending on the smoothing parameter $M$, and find the asymptotics of $\left\langle F_{M}\right\rangle_{T}$ and $\left\langle\left|F_{M}\right|^{2}\right\rangle_{T}$ as $T \rightarrow \infty$. Then we obtain the appropriate asymptotics for the original function $F(t)$ by showing that the variance of $F-F_{M}$ tends to zero as $M \rightarrow \infty$ and $T \rightarrow \infty$. This leads to Theorems 1.1 and 1.3. The proof of Theorem 1.4 is also based on the properties of the smoothed-out quantity $F_{M}$. In the process, a crucial ingredient is the asymptotics of the sum

$$
R_{\boldsymbol{\alpha}}(N)=\sum_{p=1}^{N}\left|r_{\boldsymbol{\alpha}}(p)\right|^{2}, \quad N \rightarrow \infty,
$$

which was established in [12] under the condition that $\alpha$ is Diophantine of type $\kappa<(d-1)(d-2)^{-1}$; see Proposition 2.3. We emphasize that this is the only 
reason why this condition is required in Theorems 1.3 and 1.4: the rest of the argument is independent of Diophantine properties of $\alpha$.

The authors are grateful to the referee for his remarks regarding the asymptotics of the expectation values of $F(t)$ and $S(t, \rho)$.

§2. Lattice points in a ball: proof of Theorem 1.3.

2.1. Regularized counting function. We begin by introducing a convenient regularization of the counting function $N(t)$. Using the notation $\chi(\cdot)$ for the characteristic function of the unit open ball in $\mathbb{R}^{d}$, we can write the number of lattice points $\mathbf{m} \in \mathbb{Z}^{d}$ inside the ball of radius $t>0$ (i.e. $|\mathbf{m}|<t$ ) centred at a point $\boldsymbol{\alpha} \in \mathbb{R}^{d}$ as

$$
N(t)=\sum_{\mathbf{m} \in \mathbb{Z}^{d}} \chi\left((\mathbf{m}-\boldsymbol{\alpha}) t^{-1}\right) .
$$

For $f$ in the Schwartz space $\mathrm{S}\left(\mathbb{R}^{d}\right)$, its Fourier transform is defined by

$$
\hat{f}(\mathbf{k})=\int_{\mathbb{R}^{d}} f(\mathbf{x}) \mathrm{e}^{-2 \pi i \mathbf{x} \cdot \mathbf{k}} d \mathbf{x} .
$$

To construct a regularized version of $N(t)$, let $\psi \in \mathrm{S}\left(\mathbb{R}^{d}\right)$ be a non-negative function normalized by the condition

$$
\hat{\psi}(\mathbf{0})=\int_{\mathbb{R}^{d}} \psi(\mathbf{x}) d \mathbf{x}=1 .
$$

For all $\varepsilon>0$, denote

$$
\Psi_{\varepsilon}(\mathbf{x})=\varepsilon^{-d} \psi\left(\mathbf{x} \varepsilon^{-1}\right), \quad \chi_{\varepsilon}(\mathbf{x})=\chi \star \Psi_{\varepsilon}(\mathbf{x}),
$$

and

$$
N_{M}(t)=\sum_{\mathbf{m} \in \mathbb{Z}^{d}} \chi_{\varepsilon}\left((\mathbf{m}-\boldsymbol{\alpha}) t^{-1}\right), \quad \varepsilon=\frac{1}{t M},
$$

where $M \geq 1$ is the $t$-independent parameter taken to be large later on. We assume that $\psi$ is a radial function, i.e. it depends only on $|\mathbf{x}|$, so that its Fourier transform $\hat{\psi}(\boldsymbol{\xi})$ is also radial. In a slight abuse of notation, we sometimes write $\psi(|\mathbf{x}|)$ and $\hat{\psi}(|\boldsymbol{\xi}|)$ instead of $\psi(\mathbf{x})$ and $\hat{\psi}(\boldsymbol{\xi})$. The function $\chi_{\varepsilon}$ is in $\mathbf{S}\left(\mathbb{R}^{d}\right)$ so that one can use the Poisson summation formula

$$
\sum_{\mathbf{m} \in \mathbb{Z}^{d}} f(\mathbf{m})=\sum_{\mathbf{m} \in \mathbb{Z}^{d}} \hat{f}(\mathbf{m}), \quad f \in \mathbf{S}\left(\mathbb{R}^{d}\right),
$$

to obtain

$$
\begin{aligned}
N_{M}(t) & =t^{d} \sum_{\mathbf{m} \in \mathbb{Z}^{d}} \exp (-2 \pi i \boldsymbol{\alpha} \cdot \mathbf{m}) \hat{\chi}_{\varepsilon}(t \mathbf{m}) \\
& =t^{d} \sum_{\mathbf{m} \in \mathbb{Z}^{d}} \exp (-2 \pi i \boldsymbol{\alpha} \cdot \mathbf{m}) \hat{\chi}(t \mathbf{m}) \hat{\psi}(\varepsilon t \mathbf{m}) \\
& =B_{d} t^{d}+t^{d} \sum_{\mathbf{m} \in \mathbb{Z}^{d} \backslash\{0\}} \exp (-2 \pi i \boldsymbol{\alpha} \cdot \mathbf{m}) \hat{\chi}(t \mathbf{m}) \hat{\psi}\left(|\mathbf{m}| M^{-1}\right) .
\end{aligned}
$$


The Fourier coefficients of $\chi$ can be found via the Bessel function:

$$
\begin{aligned}
\hat{\chi}(\xi) & =|\xi|^{-(d / 2)} J_{d / 2}(2 \pi|\xi|) \\
& =\sum_{l=0}^{N} P_{l}|\xi|^{-(d+1) / 2-l} \cos \left(2 \pi|\xi|+\phi_{l}\right)+O\left(|\xi|^{-(d+1) / 2-N-1}\right), \\
P_{0} & =\frac{1}{\pi}, \quad \phi_{0}=-\frac{d+1}{4} \pi,
\end{aligned}
$$

where $P_{l}$ and $\phi_{l}, l=1,2, \ldots$, are real coefficients and phases respectively, whose precise value is of no importance here. The above asymptotics are valid for all $N \geq 0$. Along with the quantity $F$ defined in (1), we define its regularization,

$$
F_{M}(t)=t^{-(d-1) / 2}\left(N_{M}(t)-B_{d} t^{d}\right) .
$$

Using (8) and (9), we represent the function $F_{M}(t)$ as a sum

$$
F_{M}(t)=\sum_{l=0}^{N} \tilde{F}_{M}^{(l)}(t)+\tilde{R}_{M}^{(N+1)}(t)
$$

where

$$
\begin{aligned}
\tilde{F}_{M}^{(l)}(t)= & P_{l} t^{-l} \sum_{\mathbf{m} \in \mathbb{Z}^{d} \backslash\{0\}} \frac{\cos \left(2 \pi t|\mathbf{m}|+\phi_{l}\right)}{|\mathbf{m}|^{(d+1) / 2+l}} \\
& \times \exp (-2 \pi i \boldsymbol{\alpha} \cdot \mathbf{m}) \hat{\psi}\left(|\mathbf{m}| M^{-1}\right), \quad l \geq 0 .
\end{aligned}
$$

Under the condition $N>(d-3) / 2$, the function $\tilde{R}_{M}^{(N+1)}(t)$ is continuous in $t>0$ and satisfies the bound

$$
\left|\tilde{R}_{M}^{(N+1)}(t)\right| \ll t^{-N-1}, \quad N>\frac{d-3}{2},
$$

uniformly in the parameter $M$. In particular, in the case $d=2$, one can simply take $N=0$. For convenience we truncate the sums $\tilde{F}_{M}^{(l)}$. Split each of them into two sums: over $|\mathbf{m}| \leq M^{1+\zeta / 2}$ and $|\mathbf{m}|>M^{1+\zeta / 2}$ with some $\zeta>0$. Since $|\hat{\psi}(\xi)| \ll(1+|\xi|)^{-H}$ with an arbitrary $H>0$, the sum over $|\mathbf{m}|>M^{1+\zeta / 2}$ is bounded by

$$
M^{H} \sum_{|\mathbf{m}| \geq M^{1+\zeta / 2}}|\mathbf{m}|^{-(d+1) / 2-l-H} \ll M^{-\zeta H / 2+d}
$$

Denote

$$
\begin{aligned}
F_{M}^{(l)}(t)= & P_{l} t^{-l} \sum_{\mathbf{m} \in \mathbb{Z}^{d} \backslash\{0\}:|\mathbf{m}| \leq M^{1+\zeta / 2}} \frac{\cos \left(2 \pi t|\mathbf{m}|+\phi_{l}\right)}{|\mathbf{m}|^{(d+1) / 2+l}} \\
& \times \exp (-2 \pi i \boldsymbol{\alpha} \cdot \mathbf{m}) \hat{\psi}\left(|\mathbf{m}| M^{-1}\right), \quad l \geq 0,
\end{aligned}
$$

and include the remaining part of the sum in the new remainder, which we denote by $R_{M}^{(N+1)}(t)$, so that

$$
F_{M}(t)=\sum_{l=0}^{N} F_{M}^{(l)}(t)+R_{M}^{(N+1)}(t)
$$


with

$$
\left|R_{M}^{(N+1)}(t)\right| \ll t^{-N-1}+M^{-H}, \quad t>0, N>\frac{d-3}{2},
$$

with an arbitrary $H>0$. Using the notation (3), we can rewrite $F_{M}^{(l)}$ in a concise form:

$$
F_{M}^{(l)}(t)=P_{l} t^{-l} \sum_{p=1}^{M^{2+\zeta}} \frac{\cos \left(2 \pi t \sqrt{p}+\phi_{l}\right)}{p^{(d+1+2 l) / 4}} r_{\alpha}(p) \hat{\psi}\left(\sqrt{p} M^{-1}\right)
$$

The proof of Theorem 1.1 relies on the following result.

THEOREM 2.1. Let $d \geq 2$, and let $\boldsymbol{\alpha} \in \mathbb{R}^{d}$ be arbitrary. Let $g \in \mathrm{C}^{\infty}(0, \infty)$ be a real-valued function such that

$$
\left|\frac{d^{m}}{d t^{m}} g(t)\right| \leq C_{m} t^{-m}, \quad t>1,
$$

for all $m=0,1, \ldots$ Then for all $l=1,2, \ldots, N$,

$$
\left|\left\langle F_{M}^{(l)}(\cdot+a) g\right\rangle_{T}\right| \ll T^{-X}
$$

for any $X>0$, uniformly in $M \geq 1$ and $a \geq 0$, with the implied constant which depends only on the constants $C_{m}$ in (13).

The main ingredient in the proof of Theorems 1.3 and 1.4 is the following theorem.

THEOREM 2.2. Let $\boldsymbol{\alpha}$ be as in Theorem 1.3, and let $\mathfrak{A}_{\alpha}$ be as defined in (4). Suppose that $M \leq T^{H}$ with some $H>0$. Then $\left\langle\left|F_{M}^{(l)}\right|^{2}\right\rangle_{T} \ll T^{-2 l}$, for all $l \geq 1$, and

$$
\left\langle\left|F_{M}^{(0)}\right|^{2}\right\rangle_{T} \rightarrow \frac{1}{2 \pi^{2}} \mathfrak{A}_{\alpha}, \quad M \rightarrow \infty .
$$

The proof of Theorem 2.1 is elementary and is given next.

2.2. Proof of Theorem 2.1. Compute the expectation value of $F_{M}^{(l)}(\cdot+a) g$ :

$$
\begin{aligned}
\left\langle F_{M}^{(l)}(\cdot+a) g\right\rangle_{T} & =\frac{1}{T} \int_{0}^{\infty} F_{M}^{(l)}(t+a) g(t) \omega\left(t T^{-1}\right) d t \\
= & T^{-l} P_{l} \sum_{p=1}^{M^{2+\zeta}} \frac{\hat{\psi}\left(\sqrt{p} M^{-1}\right)}{p^{(d+1+2 l) / 4}} r_{\alpha}(p) \int_{0}^{\infty} \cos \left(2 \pi(T t+a) \sqrt{p}+\phi_{l}\right) \\
& \times \omega(t)\left(t+a T^{-1}\right)^{-l} g(T t) d t \\
= & T^{-l} P_{l} \sum_{p=1}^{M^{2+\zeta}} \frac{\hat{\psi}\left(\sqrt{p} M^{-1}\right)}{p^{(d+1+2 l) / 4}} r_{\alpha}(p) \operatorname{Re}\left(\exp \left(-i \phi_{l}-i 2 \pi a \sqrt{p}\right) \hat{h}(T \sqrt{p} ; T)\right),
\end{aligned}
$$

where we have denoted $h(t ; T)=\omega(t)\left(t+a T^{-1}\right)^{-l} g(T t)$. In view of (13), the Fourier transform $\hat{h}(s ; T)$ satisfies the bound $|\hat{h}(s ; T)| \ll(1+|s|)^{-X}$ for 
any $X>0$ uniformly in $T>0$. Thus we have $|\hat{h}(T \sqrt{p} ; T)| \ll T^{-X} p^{-X / 2}$, and hence

$$
\left|\left\langle F_{M}^{(l)}(\cdot+a) g\right\rangle_{T}\right| \ll T^{-l-X} \sum_{p=1}^{\infty}\left|r_{\alpha}(p)\right| p^{-X / 2} .
$$

Estimating $\left|r_{\alpha}(p)\right| \ll p^{d / 2}$ and taking a sufficiently large $X$, we arrive at the required estimate.

For Theorem 2.2 we need more information about the sum (7).

2.3. Properties of $r_{\alpha}(n)$. The proof of Theorem 2.2 relies on the following delicate asymptotic result for $R_{\boldsymbol{\alpha}}(N)$ (see (7)), established in [12].

PROPOSITION 2.3. Let the components of $(\alpha, 1)$ be linearly independent over $\mathbb{Q}$, and let $\alpha$ be Diophantine of type $\kappa<(d-1)(d-2)^{-1}$. Then

$$
\lim _{N \rightarrow \infty} \frac{1}{N^{d / 2}} R_{\alpha}(N)=B_{d}
$$

For $d=2$, the above asymptotic formula was proved for Diophantine $\boldsymbol{\alpha}$ in [4]. The estimate of the form $R_{\boldsymbol{\alpha}}(N) \ll N^{d / 2+\epsilon}$ was announced in [1] under a stronger condition that $\boldsymbol{\alpha}$ should be multiplicatively Diophantine. We refer to [12] for further comparison and more detailed discussion.

Note that for the proof of Theorem 1.3, it would suffice to know the asymptotic estimate $R_{\alpha}(N) \ll N^{d / 2}$. On the other hand, Theorem 1.4 hinges on the asymptotic formula (14). We reiterate that Proposition 2.3 is the only ingredient of our proof that necessitates the Diophantine properties of $\boldsymbol{\alpha}$. Otherwise our argument goes through for arbitrary $\boldsymbol{\alpha}$.

Proposition 2.3 has a number of useful elementary consequences which we gather below.

LEMMA 2.4. Assume that $R_{\boldsymbol{\alpha}}(n) \ll n^{b}$ with some $b>0$. Then for any $1 \leq N_{1} \leq N_{2}<\infty$ and any $\beta \in \mathbb{R}$, we have

$$
\sum_{p=N_{1}}^{N_{2}} \frac{\left|r_{\alpha}(p)\right|^{2}}{p^{\beta}} \ll \begin{cases}N_{1}^{b-\beta}+N_{2}^{b-\beta}, & b \neq \beta, \\ \log N_{1}+\log N_{2}, & b=\beta .\end{cases}
$$

Proof. Using the "summation by parts" formula

$$
\begin{aligned}
& \sum_{n=N_{1}}^{N_{2}}(f(n+1)-f(n)) g(n) \\
& \quad=-\sum_{n=N_{1}+1}^{N_{2}} f(n)(g(n)-g(n-1))+f\left(N_{2}+1\right) g\left(N_{2}\right)-f\left(N_{1}\right) g\left(N_{1}\right),
\end{aligned}
$$


with $f(n)=R_{\boldsymbol{\alpha}}(n)$ and $g(n)=(n+1)^{-\beta}$, we get

$$
\begin{aligned}
\sum_{p=N_{1}}^{N_{2}} \frac{\left|r_{\alpha}(p)\right|^{2}}{p^{\beta}}= & \sum_{p=N_{1}-1}^{N_{2}-1}(f(p+1)-f(p)) g(p) \\
= & -\sum_{p=N_{1}}^{N_{2}-1} R_{\boldsymbol{\alpha}}(p)\left((p+1)^{-\beta}-p^{-\beta}\right) \\
& +R_{\boldsymbol{\alpha}}\left(N_{2}\right) N_{2}^{-\beta}-R_{\boldsymbol{\alpha}}\left(N_{1}-1\right) N_{1}^{-\beta}
\end{aligned}
$$

Now the required estimates follow.

By virtue of Proposition 2.3, Lemma 2.4 implies that the series (4) converges. The following lemma will be used in $\S 3$.

Lemma 2.5. Suppose that $\boldsymbol{\alpha}$ satisfies the conditions of Proposition 2.3. Then for any positive numbers $L_{1}$ and $L_{2}$ with $L_{1}<L_{2}$,

$$
\lim _{\rho \rightarrow 0} \rho^{d} \sum_{L_{1} \leq \rho^{2} p<L_{2}}\left|r_{\alpha}(p)\right|^{2}=B_{d} L_{2}^{d / 2}-B_{d} L_{1}^{d / 2} .
$$

Proof. Let us find an upper bound:

$$
\begin{aligned}
& \limsup _{\rho \rightarrow 0} \rho^{d} \sum_{L_{1} \leq \rho^{2} p<L_{2}}\left|r_{\boldsymbol{\alpha}}(p)\right|^{2} \\
& \quad \leq \lim _{\rho \rightarrow 0}\left(\rho^{d} R_{\boldsymbol{\alpha}}\left(\left[L_{2} \rho^{-2}\right]+1\right)-\rho^{d} R_{\boldsymbol{\alpha}}\left(\left[L_{1} \rho^{-2}\right]\right)\right) \\
& \quad=B_{d} L_{2}^{d / 2}-B_{d} L_{1}^{d / 2} .
\end{aligned}
$$

Similarly the lower bound follows.

2.4. Proof of Theorem 2.2. Before proceeding to the proof, we write out the $F_{M}^{(l)}$-variance:

$$
\begin{aligned}
\left\langle\left|F_{M}^{(l)}\right|^{2}\right\rangle_{T}= & \frac{1}{T} \int_{0}^{\infty}\left|F_{M}^{(l)}(t)\right|^{2} \omega\left(t T^{-1}\right) d t \\
= & T^{-2 l} P_{l}^{2} \sum_{p, q=1}^{M^{2+\zeta}} \frac{\hat{\psi}\left(\sqrt{p} M^{-1}\right) \overline{\hat{\psi}\left(\sqrt{q} M^{-1}\right)}}{(p q)^{(d+1+2 l) / 4}} r_{\boldsymbol{\alpha}}(p) \overline{r_{\boldsymbol{\alpha}}(q)} \\
& \times \int_{0}^{\infty} \cos \left(2 \pi t \sqrt{p}+\phi_{l}\right) \cos \left(2 \pi t \sqrt{q}+\phi_{l}\right) \\
& \times \omega\left(t T^{-1}\right)\left(t T^{-1}\right)^{-2 l} \frac{1}{T} d t .
\end{aligned}
$$

The integral on the right-hand side equals

$$
\frac{1}{2} \operatorname{Re}\left(\mathrm{e}^{-2 i \phi_{l}} \hat{\omega}_{l}(T(\sqrt{p}+\sqrt{q}))\right)+\frac{1}{2} \operatorname{Re}\left(\hat{\omega}_{l}(T(\sqrt{p}-\sqrt{q}))\right),
$$

where we have denoted $\omega_{l}(t)=\omega(t) t^{-2 l}$. As in the proof of Theorem 2.1 above, the Fourier transform $\hat{\omega}_{l}(s)$ satisfies the bound $\left|\hat{\omega}_{l}(s)\right| \ll(1+|s|)^{-X}$ for 
any $X>0$. Thus $\left|\hat{\omega}_{l}(T(\sqrt{p}+\sqrt{q}))\right| \ll T^{-X} p^{-X / 4} q^{-X / 4}$, and hence the terms containing $\hat{\omega}_{l}(T(\sqrt{p}+\sqrt{q}))$ are bounded by

$$
T^{-2 l-X} \sum_{p} \frac{\left|r_{\boldsymbol{\alpha}}(p)\right|^{2}}{p^{(d+1+2 l) / 2+X / 4}} \sum_{q} \frac{1}{q^{X / 4}} .
$$

By Proposition 2.3 and Lemma 2.4, this does not exceed $T^{-2 l-X}$. Thus these terms give a negligible contribution to $\left\langle\left|F_{M}^{(l)}\right|^{2}\right\rangle_{T}$.

Let us concentrate on the terms containing $\hat{\omega}_{l}(T(\sqrt{p}-\sqrt{q}))$. This sum splits in two parts: diagonal, that is the sum with $p=q$,

$$
D^{(l)}(M, T)=\frac{\hat{\omega}_{l}(0)}{2} P_{l}^{2} T^{-2 l} \sum_{p=1}^{M^{2+\zeta}} \frac{\left|\hat{\psi}\left(\sqrt{p} M^{-1}\right)\right|^{2}}{p^{(d+1) / 2+l}}\left|r_{\alpha}(p)\right|^{2},
$$

and off-diagonal, that is the sum with $p \neq q$,

$$
\begin{aligned}
K^{(l)}(M, T)= & \frac{1}{2} P_{l}^{2} T^{-2 l} \sum_{\substack{p, q=1 \\
p \neq q}}^{M^{2+\zeta}} \frac{\hat{\psi}\left(\sqrt{p} M^{-1}\right) \overline{\hat{\psi}\left(\sqrt{q} M^{-1}\right)}}{(p q)^{(d+1+2 l) / 4}} \\
& \times r_{\alpha}(p) \overline{r_{\alpha}(q)} \operatorname{Re}\left(\hat{\omega}_{l}(T(\sqrt{p}-\sqrt{q}))\right) .
\end{aligned}
$$

Note that $D^{(0)}$ depends only on $M$ and thus we write $D^{(0)}(M)$.

Let us investigate the quantities (16) and (17).

LEMMA 2.6 (Diagonal part). Under the conditions of Theorem 1.3, we have

$$
\lim _{M \rightarrow \infty} D^{(0)}(M)=\frac{1}{2 \pi^{2}} \mathfrak{A}_{\alpha},
$$

and

$$
D^{(l)}(M, T) \ll T^{-2 l}, \quad l \geq 1 .
$$

Proof. The estimate (19) follows from the boundedness of the function $\hat{\psi}$ and from Lemma 2.4. To prove (18), recall that $\left|\hat{\psi}\left(\sqrt{p} M^{-1}\right)\right|^{2} \leq|\hat{\psi}(0)|^{2}=1$, $\hat{\omega}(0)=1$ and $P_{0}^{2}=1 / \pi^{2}$ so that, by the Lebesgue convergence theorem, the result for $D^{(0)}$ follows.

LEMMA 2.7 (Off-diagonal part). Under the conditions of Theorem 1.3 and the assumption that $M \leq T^{H}$ for some $H>0$, we have

$$
K^{(l)}(M, T) \ll T^{-\eta-2 l}, \quad l \geq 0,
$$

for any $\eta \in(0,1)$.

Proof. Fix the index $l$. By Hölder's inequality,

$$
\left|K^{(l)}(M, T)\right| \ll T^{-2 l} \sum_{\substack{p, q=1 \\ p \neq q}}^{M^{2+\zeta}} \frac{\left|\hat{\psi}\left(\sqrt{p} M^{-1}\right)\right|^{2}}{p^{(d+1) / 2+l}}\left|r_{\alpha}(p)\right|^{2}|\hat{\omega}(T(\sqrt{p}-\sqrt{q}))| .
$$


Split the sum on the right-hand side into two sums: $K_{1}=K_{1}(M, T)$ for $|\sqrt{p}-\sqrt{q}| \geq T^{-\eta}, \quad \eta \in(0,1)$, and $K_{2}=K_{2}(M, T)$ for $|\sqrt{p}-\sqrt{q}|<T^{-\eta}$. Since $|\hat{\omega}(s)| \ll|s|^{-X}$ with any $X>0$, we have

$$
\begin{aligned}
K_{1} & \ll M^{2+\zeta} T^{-X(1-\eta)-2 l} \sum_{p=1}^{M^{2+\zeta}} p^{-(d+1) / 2-l}\left|r_{\alpha}(p)\right|^{2} \\
& \leq M^{2+\zeta} T^{-X(1-\eta)-2 l} R_{\alpha}\left(M^{2+\zeta}\right) .
\end{aligned}
$$

In view of (14), this implies that

$$
K_{1} \ll M^{(2+\zeta)((d / 2)+1)} T^{-X(1-\eta)-2 l} .
$$

As $M \leq T^{H}$, choosing $X$ sufficiently large, one obtains that $K_{1} \ll T^{-\eta-2 l}$.

To estimate the sum $K_{2}$, note that for $p$ and $q$ satisfying $0<|\sqrt{p}-\sqrt{q}|<$ $T^{-\eta}$, we have

$$
0<|\sqrt{p}-\sqrt{q}|=\frac{|p-q|}{\sqrt{p}+\sqrt{q}}<T^{-\eta},
$$

so that

$$
0<|p-q|<T^{-\eta}(\sqrt{p}+\sqrt{q})<3 \sqrt{p} T^{-\eta} .
$$

Thus, for each $p$, there are at most $6 \sqrt{p} T^{-\eta}$ of $q$ s contributing to the sum $K_{2}$. Thus, according to (14) and Lemma 2.4,

$$
K_{2} \ll T^{-\eta-2 l} \sum_{p=1}^{M^{2+\zeta}}\left|r_{\boldsymbol{\alpha}}(p)\right|^{2} p^{-(d / 2)-l} \ll \begin{cases}T^{-\eta} \log M, & l=0, \\ T^{-\eta-2 l}, & l \geq 1 .\end{cases}
$$

Modifying $\eta$ suitably, one concludes that $K_{2} \ll T^{-\eta-2 l}$ for all $l \geq 0$ and any $\eta \in(0,1)$.

Proof of Theorem 2.2. Combine Lemmas 2.6 and 2.7.

2.5. Proof of Theorems 1.1 and 1.3. The final step of the proof of Theorems 1.1 and 1.3 is to show that $F_{M}(t)$ is a good approximation of $F(t)$. Now we make one further assumption on the function $\psi$. Namely, we suppose that $\psi$ has support in the unit ball $\{\mathbf{x}:|\mathbf{x}| \leq 1\}$ so that

$$
\begin{aligned}
\left|N(t)-N_{M}(t)\right| & =\left|\sum_{\mathbf{m}:|| \mathbf{m}-\boldsymbol{\alpha}|-t| \leq M^{-1}}\left(\chi\left((\mathbf{m}-\boldsymbol{\alpha}) t^{-1}\right)-\chi_{\varepsilon}\left((\mathbf{m}-\boldsymbol{\alpha}) t^{-1}\right)\right)\right| \\
& \leq \sum_{\mathbf{m}:|\mathbf{m}-\boldsymbol{\alpha}|-t \mid \leq M^{-1}} 1,
\end{aligned}
$$

with $\varepsilon=(t M)^{-1}$.

Lemma 2.8. Let $\psi$ be as above. Then for any $\boldsymbol{\alpha} \in \mathbb{R}^{d}$, and any $T \geq 1$, $M \geq 1$, we have

$$
\left\langle\left|F(\cdot+a)-F_{M}(\cdot+a)\right|^{2}\right\rangle_{T} \ll T^{d-1} M^{-1},
$$

uniformly in a such that $0 \leq a \ll T$. 
Proof. Let $\mathbf{x}^{\prime}=\mathbf{x}-\boldsymbol{\alpha}$ for $\mathbf{x} \in \mathbb{R}^{d}$. It follows from (22) that

$$
\left|F(t+a)-F_{M}(t+a)\right| \leq t^{(1-d) / 2} \sum_{\mathbf{m}:|| \mathbf{m}^{\prime}|-(t+a)| \leq M^{-1}} 1 .
$$

Since the function $\omega$ is supported on the positive semi-axis $t \geq t_{0}$, we have

$$
\begin{aligned}
\left\langle\left|F(t+a)-F_{M}(t+a)\right|^{2}\right\rangle_{T} & \ll \frac{1}{T^{d}} \int_{0}^{\infty}\left(\sum_{\substack{\mathbf{m}:|| \mathbf{m}^{\prime}|-(t+a)| \leq M^{-1}, \mathbf{n}:|| \mathbf{n}^{\prime}|-(t+a)| \leq M^{-1}}} 1\right) \omega\left(\frac{t}{T}\right) d t \\
& \ll \frac{1}{T^{d}} \sum_{\substack{\mathbf{m}:\left|\mathbf{m}^{\prime}\right| \ll T, \mathbf{n}:|| \mathbf{m}^{\prime}|-| \mathbf{n}^{\prime}||<1}} \int_{|| \mathbf{m}^{\prime}|-(t+a)| \leq M^{-1}} d t \\
& \ll \frac{1}{T^{d} M} \sum_{\substack{\mathbf{m}:\left|\mathbf{m}^{\prime}\right| \ll T, \mathbf{n}:|| \mathbf{m}^{\prime}|-| \mathbf{n}^{\prime}||<1}} 1 \ll \frac{T^{d-1}}{M} .
\end{aligned}
$$

The estimate is proved.

Proof of Theorem 1.1. Let $g$ be a function as in Theorem 2.1, and let $a \geq 0$ be some number. By (10),

$$
\left\langle F_{M}(\cdot+a) g\right\rangle_{T}=\sum_{l=1}^{N}\left\langle F_{M}^{(l)}(\cdot+a) g\right\rangle_{T}+\left\langle R_{M}^{(N+1)}(\cdot+a) g\right\rangle_{T} .
$$

Assuming that $M=T^{Y}$ with some $Y>0$, and choosing sufficiently large $N$, we obtain from (11) and Theorem 2.1 that

$$
\left|\left\langle F_{M}(\cdot+a) g\right\rangle_{T}\right| \ll T^{-X},
$$

for any $X>0$, uniformly in $a \geq 0$. On the other hand, due to Lemma 2.8, we have

$$
\left\langle\left|F(\cdot+a)-F_{M}(\cdot+a)\right| g\right\rangle_{T}^{2} \ll\left\langle\left|F(\cdot+a)-F_{M}(\cdot+a)\right|^{2}\right\rangle_{T} \ll T^{d-1-Y},
$$

uniformly in $a$ such that $0 \leq a \ll T$. Since $Y>0$ is arbitrary, this implies that

$$
\left|\langle F(\cdot+a) g\rangle_{T}\right| \ll T^{-X},
$$

for any $X>0$ uniformly in $a$. Taking $a=0, g(t)=1$ proves part 1 of Theorem 1.1.

In order to prove part 2, rewrite (2) in the following form:

$$
S(t, \rho)=\frac{1}{\sqrt{\rho}}\left(1+\rho t^{-1}\right)^{(d-1) / 2} F(t+\rho)-\frac{1}{\sqrt{\rho}} F(t),
$$

and assume that $T^{-Z}<\rho \ll 1$ with some $Z>0$. In view of (23) with $a=\rho$ and $g(t)=1$, the second term on the right-hand side of (24) tends to zero as $T \rightarrow \infty$. For the first term we use (23) with $a=\rho$ and $g(t)=\left(1+\rho t^{-1}\right)^{(d-1) / 2}$. This is legitimate since this choice of the function $g$ satisfies (13) for $\rho \ll 1$. This completes the proof of part 2. 
Proof of Theorem 1.3. Choose the parameter $M$ to satisfy the conditions $M \leq T^{H}$, with some $H>0$, and $M T^{1-d} \rightarrow \infty$ as $T \rightarrow \infty$. For instance, one can take $M=T^{d}$.

Denoting $\|f\|=\sqrt{\left\langle|f|^{2}\right\rangle_{T}}$, we have, by (10),

$$
\left\|F_{M}\right\|-\left\|F_{M}^{(0)}\right\| \mid \leq \sum_{l=1}^{N}\left\|F_{M}^{(l)}\right\|+\left\|R_{M}^{(N+1)}\right\| .
$$

In view of (11) and Theorem 2.2, the above inequality leads to the asymptotics

$$
\left\|F_{M}\right\|^{2} \rightarrow \frac{1}{2 \pi^{2}} \mathfrak{A}_{\alpha}, \quad T \rightarrow \infty
$$

Theorem 1.3 now follows from the inequality $\left|\|F\|-\left\|F_{M}\right\|\right| \leq\left\|F-F_{M}\right\|$ and Lemma 2.8 since $M T^{1-d} \rightarrow \infty$.

To conclude this section we make a remark of a technical nature. As we have already mentioned in the introduction, our proof follows the standard strategy, that is, we study first the smoothed-out quantity $F_{M}$, after which we return to the initial $F(t)$ by proving that $F_{M}(t)-F(t)$ is small in the appropriate sense. For lattice counting problems in general, in order to implement this plan, one usually needs to make a very careful choice of the smoothing parameter (which is $M$ in this paper), to ensure that the smoothed-out quantity admits an asymptotic formula, and at the same time does not differ too much from the unsmoothed one. These two requirements usually impose quite tight upper and lower bounds on the smoothing parameter: see, for example [3, 5, 7]. This necessitates more precise bounds at various steps of the proof. For instance, in $[3,5]$, the unsmoothing part of the proof (Lemma 2.8 in this paper) requires subtle estimates for close pairs of lattice points. In the present paper, however, the choice of $M$ is virtually unrestricted (see Theorem 2.2), the unsmoothing argument is quite elementary, and a very crude estimate for the close pairs suffices (see Lemma 2.8). The explanation of this freedom of choice of $M$ lies with the asymptotics (14) of the function $R_{\alpha}(N)$ for Diophantine $\alpha$ s. A naive upper bound for $R_{\alpha}(N)$, obtained by estimating $r_{\alpha}(p)$ via the number of lattice points on the sphere of radius $\sqrt{p}$, would not be sufficient.

§3. Lattice points in a thin shell: proof of Theorem 1.4.

3.1. Regularized counting function. Rewrite (24) as

$$
S(t, \rho)=\frac{F(t+\rho)-F(t)}{\sqrt{\rho}}-\frac{1}{\sqrt{\rho}}\left(1-(t+\rho)^{(d-1) / 2} t^{-(d-1) / 2}\right) F(t+\rho) .
$$

We make an elementary observation concerning the shifted quantities of the form $F(t+\rho)$ and others. Recalling that $\omega(t)=0$ outside some interval $\left[t_{0}, t_{1}\right]$ where $0<t_{0}<t_{1}<\infty$, define a non-negative function $v \in \mathrm{C}_{0}^{\infty}(\mathbb{R})$ such that

$$
\widetilde{v}(t)= \begin{cases}\max _{t} \omega(t), & t \in\left[t_{0} / 2,2 t_{1}\right] \\ 0, & t \leq t_{0} / 4\end{cases}
$$


Then for any function $f$, assuming that $\rho$ is sufficiently small, we have

$$
\langle|f(\cdot+\rho)|\rangle_{\omega, T} \leq\|\widetilde{v}\|_{\mathrm{L}^{1}}\langle|f|\rangle_{\nu, T}, \quad v=\frac{\widetilde{v}}{\|\widetilde{v}\|_{\mathrm{L}^{1}}} .
$$

The next lemma reduces the study of $S(\cdot, \rho)$ to finding the asymptotics of a smoothed-out quantity

$$
S(t, \rho ; M):=\frac{F_{M}^{(0)}(t+\rho)-F_{M}^{(0)}(t)}{\sqrt{\rho}} .
$$

Lemma 3.1. Let $\boldsymbol{\alpha}$ be as in Theorem 1.4. Let $M=T^{d}$ and $\rho \gg T^{-\sigma}$ with some $\sigma \in(0,1)$. Then

$$
\left\langle|S(\cdot, \rho)-S(\cdot, \rho ; M)|^{2}\right\rangle_{T} \rightarrow 0, \quad \rho \rightarrow 0 .
$$

Proof. As before, denote $\|f\|=\sqrt{\left\langle|f|^{2}\right\rangle_{T}}$. Moreover, for any functions $f$ and $g$ depending on $\rho, M$ and $T$, we write $f \asymp g$ if $\|f-g\| \rightarrow 0$ as $\rho \rightarrow 0$, $M \rightarrow \infty$ and $T \rightarrow \infty$.

Consider each term in (25) separately. The average of the square of the last summand in (25) is bounded by

$$
\rho T^{-2}\|F(\cdot+\rho)\|^{2},
$$

which, by Theorem 1.3 and observation (26), tends to zero as $\rho \rightarrow 0$, so that

$$
S(t, \rho) \asymp \frac{F(t+\rho)-F(t)}{\sqrt{\rho}} .
$$

Now consider the first term on the right-hand side of (25). Since $M=T^{d}$, it follows from Lemma 2.8 that

$$
\frac{1}{\rho}\left\|F-F_{M}\right\|^{2} \ll \rho^{-1} T^{-1} \ll T^{\sigma-1} \rightarrow 0, \quad T \rightarrow \infty,
$$

and the same bound holds for the difference $F(\cdot+\rho)-F_{M}(\cdot+\rho)$. This shows that

$$
S(t, \rho) \asymp \frac{F_{M}(t+\rho)-F_{M}(t)}{\sqrt{\rho}} .
$$

We now estimate the contribution of each summand in (10). First of all, $R_{M}^{(N+1)} \asymp 0$ in view of (11). Furthermore, by Theorem 2.2,

$$
\rho^{-1}\left\|F_{M}^{(l)}(\cdot)\right\|^{2}+\rho^{-1}\left\|F_{M}^{(l)}(\cdot+\rho)\right\|^{2} \ll \rho^{-1} T^{-2 l} \ll T^{-1}, \quad l \geq 1,
$$

so that

$$
S(t, \rho) \asymp \frac{F_{M}^{(0)}(t+\rho)-F_{M}^{(0)}(t)}{\sqrt{\rho}},
$$

as required. 
3.2. Proof of Theorem 1.4. Now we compute $\left\langle|S(\cdot, \rho ; M)|^{2}\right\rangle_{T}$. From (12) we have

$$
\begin{aligned}
F_{M}^{(0)}(t+\rho)-F_{M}^{(0)}(t) \\
=\frac{1}{\pi} \sum_{p=1}^{M^{2+\zeta}}\left(\cos \left(2 \pi(t+\rho) \sqrt{p}+\phi_{0}\right)-\cos \left(2 \pi t \sqrt{p}+\phi_{0}\right)\right) \\
\quad \times \frac{r_{\alpha}(p)}{p^{(d+1) / 4}} \hat{\psi}\left(\sqrt{p} M^{-1}\right) \\
=-\frac{2}{\pi} \sum_{p=1}^{M^{2+\zeta}} \sin \left(2 \pi(t+\rho / 2) \sqrt{p}+\phi_{0}\right) \sin (\pi \rho \sqrt{p}) \frac{r_{\alpha}(p)}{p^{(d+1) / 4}} \hat{\psi}\left(\sqrt{p} M^{-1}\right),
\end{aligned}
$$

where $\phi_{0}$ is defined in (9). Therefore

$$
\begin{aligned}
\left\langle|S(\cdot, \rho ; M)|^{2}\right\rangle_{T} & \\
= & \rho^{-1} \pi^{-2} \sum_{1 \leq p, q \leq M^{2+\zeta}} \sin (\pi \rho \sqrt{p}) \sin (\pi \rho \sqrt{q}) \\
& \times r_{\alpha}(p) \overline{r_{\alpha}(q)} \hat{\psi}\left(\sqrt{p} M^{-1}\right) \overline{\hat{\psi}\left(\sqrt{q} M^{-1}\right)}(p q)^{-(d+1) / 4} \\
& \times\left\langle\sin \left(2 \pi\left(t+\frac{\rho}{2}\right) \sqrt{p}+\phi_{0}\right) \sin \left(2 \pi\left(t+\frac{\rho}{2}\right) \sqrt{q}+\phi_{0}\right)\right\rangle_{T} .
\end{aligned}
$$

The average $\langle\cdot\rangle_{T}$ on the right-hand side equals

$$
\begin{aligned}
& -\frac{1}{2} \operatorname{Re}\left(\exp \left(i 2 \phi_{0}+i \pi \rho(\sqrt{p}+\sqrt{q})\right) \hat{\omega}(-T(\sqrt{p}+\sqrt{q}))\right) \\
& +\frac{1}{2} \operatorname{Re}(\exp (-i \pi \rho(\sqrt{p}-\sqrt{q})) \hat{\omega}(T(\sqrt{p}-\sqrt{q}))) .
\end{aligned}
$$

As in the proof of Theorem 2.2, by the fast decay of $|\hat{\omega}|$, the first term in (27) makes a small contribution as $T \rightarrow \infty$. The remaining sum splits into two parts, $\left\langle|S(\cdot, \rho ; M)|^{2}\right\rangle_{T}=D(\rho, M)+K(\rho, M, T)$, where $D(\rho, M)$ and $K(\rho, M, T)$ are the diagonal part and the off-diagonal part, respectively:

$$
D(\rho, M)=\frac{2}{\rho \pi^{2}} \sum_{1 \leq p \leq M^{2+\zeta}} \sin ^{2}(\pi \rho \sqrt{p})\left|r_{\alpha}(p)\right|^{2}\left|\hat{\psi}\left(\sqrt{p} M^{-1}\right)\right|^{2} p^{-(d+1) / 2}
$$

and

$$
\begin{aligned}
K(\rho, M, T)= & \frac{2}{\rho \pi^{2}} \sum_{\substack{p, q=1 \\
p \neq q}}^{M^{2+\zeta}} \frac{\hat{\psi}\left(\sqrt{p} M^{-1}\right) \overline{\hat{\psi}\left(\sqrt{q} M^{-1}\right)}}{(p q)^{(d+1) / 4}} r_{\alpha}(p) \overline{r_{\alpha}(q)} \\
& \times \sin (\pi \rho \sqrt{p}) \sin (\pi \rho \sqrt{q}) \\
& \times \operatorname{Re}(\exp (-i \pi \rho(\sqrt{p}-\sqrt{q})) \hat{\omega}(T(\sqrt{p}-\sqrt{q}))) .
\end{aligned}
$$

As in the proof of Theorem 1.3, we show that the off-diagonal part $K(\rho, M, T)$ does not contribute to the asymptotics, and explicitly compute the asymptotics of the diagonal part $D(\rho, M)$. 
LEMma 3.2. Suppose that $\boldsymbol{\alpha}$ is Diophantine of type $\kappa<(d-1)(d-2)^{-1}$ and the components of $(\alpha, 1)$ are linearly independent over $\mathbb{Q}$. Then

$$
D(\rho, M) \rightarrow d B_{d}
$$

as $\rho \rightarrow 0$ and $M \rho \rightarrow \infty$.

LEMma 3.3. Suppose that $\boldsymbol{\alpha}$ is Diophantine of type $\kappa<(d-1)(d-2)^{-1}$ and the components of $(\alpha, 1)$ are linearly independent over $\mathbb{Q}$. If $M \leq T^{H}$ with some $H>0$, then

$$
K(\rho, M, T) \ll \rho^{-1} T^{-\eta},
$$

for all $\rho>0$ and any $\eta \in(0,1)$.

Proof of Lemma 3.3. By Hölder's inequality $\rho K(\rho, M, T)$ satisfies the bound (21) with $l=0$, and hence $\rho K(\rho, M, T)$ satisfies the estimate (20) with $l=0$. Thus $\rho K(\rho, M, T) \ll T^{-\eta}$ for any $\eta \in(0,1)$, as required.

3.2.1. Proof of Lemma 3.2. Let $\epsilon$ and $L$ be some fixed number with $0<\epsilon<$ $L<\infty$ and split the sum (28) into three components:

$$
\begin{aligned}
& S_{<}(\epsilon ; \rho)=\sum_{1 \leq p<\epsilon \rho^{-2}} \cdots, \quad \tilde{S}_{0}(\epsilon, L ; \rho)=\sum_{\epsilon \rho^{-2} \leq p<L \rho^{-2}} \cdots, \\
& S_{>}(L ; \rho)=\sum_{L \rho^{-2} \leq p \leq M^{2+\zeta}} \cdots .
\end{aligned}
$$

Estimating $\sin ^{2}(\pi \rho \sqrt{p}) \leq \pi^{2} \rho^{2} p$, we deduce from Proposition 2.3 and Lemma 2.4 that

$$
S_{<}(\epsilon ; \rho) \ll \rho \sum_{1 \leq p<\epsilon \rho^{-2}}\left|r_{\alpha}(p)\right|^{2} p^{-(d-1) / 2} \ll \sqrt{\epsilon} .
$$

Estimating $|\sin (\pi \rho \sqrt{p})|$ by 1 , we also get

$$
S_{>}(L ; \rho) \ll \rho^{-1} \sum_{L \rho^{-2} \leq p \leq M^{2+\zeta}}\left|r_{\boldsymbol{\alpha}}(p)\right|^{2} p^{-(d+1) / 2} \ll L^{-1 / 2} .
$$

Now compare $\tilde{S}_{0}(\epsilon, L ; \rho)$ with

$$
S_{0}(\epsilon, L ; \rho)=2 \rho^{-1} \pi^{-2} \sum_{\epsilon \rho^{-2} \leq p<L \rho^{-2}} \sin ^{2}(\pi \rho \sqrt{p})\left|r_{\alpha}(p)\right|^{2} p^{-(d+1) / 2} .
$$

Recall that $|\hat{\psi}(0)|=1$. Since

$$
\sup _{\epsilon \rho^{-2} \leq p \leq L \rho^{-2}}\left|\hat{\psi}\left(\sqrt{p} M^{-1}\right)-\hat{\psi}(0)\right| \ll \sqrt{L} \rho^{-1} M^{-1},
$$

we have $\tilde{S}_{0}(\epsilon, L ; \rho)-S_{0}(\epsilon, L ; \rho) \rightarrow 0$ as $M \rho \rightarrow \infty$.

Let us concentrate on the asymptotics of $S_{0}$. 
LEMma 3.4. Suppose that $\boldsymbol{\alpha}$ is Diophantine of type $\kappa<(d-1)(d-2)^{-1}$ and the components of $(\boldsymbol{\alpha}, 1)$ are linearly independent over $\mathbb{Q}$. Then for any $\epsilon$ and $L$ where $0<\epsilon<L<\infty$, the sum $S_{0}$, defined in (32), satisfies

$$
\lim _{\rho \rightarrow 0} S_{0}(\epsilon, L ; \rho)=\frac{d B_{d}}{\pi^{2}} \int_{\epsilon}^{L} \frac{\sin ^{2}(\pi \sqrt{t})}{t^{3 / 2}} d t .
$$

Proof. Partition the interval $[\epsilon, L)$ into $A$ equal intervals of length $\delta=$ $(L-\epsilon) A^{-1}$, and divide $S_{0}$ into $A$ corresponding sub-sums of the form

$$
\begin{gathered}
S_{0}^{(k)}(\epsilon, L ; \rho)=2 \rho^{-1} \pi^{-2} \sum_{\epsilon+\delta k \leq \rho^{2} p<\epsilon+\delta(k+1)} \sin ^{2}(\pi \rho \sqrt{p})\left|r_{\alpha}(p)\right|^{2} p^{-(d+1) / 2}, \\
k=0,1, \ldots, A-1 .
\end{gathered}
$$

With the notation

$$
U(t)=t^{-(d+1) / 2} \sin ^{2}(\pi \sqrt{t}), \quad t>0,
$$

rewrite

$$
S_{0}^{(k)}(\epsilon, L ; \rho)=2 \pi^{-2} \rho^{d} \sum_{\epsilon+\delta k \leq \rho^{2} p<\epsilon+\delta(k+1)} U\left(\rho^{2} p\right)\left|r_{\boldsymbol{\alpha}}(p)\right|^{2} .
$$

Since

$$
\left|U^{\prime}(t)\right| \ll t^{-(d+2) / 2}, \quad t>0
$$

we have

$$
\begin{gathered}
\left.\left|S_{0}^{(k)}(\epsilon, L ; \rho)-2 \pi^{-2} \rho^{d} U(\epsilon+\delta k) \sum_{\epsilon+\delta k \leq \rho^{2} p<\epsilon+\delta(k+1)}\right| r_{\boldsymbol{\alpha}}(p)\right|^{2} \mid \\
\ll \rho^{d} \delta \epsilon^{-(d+2) / 2} \sum_{\epsilon+\delta k \leq \rho^{2} p<\epsilon+\delta(k+1)}\left|r_{\boldsymbol{\alpha}}(p)\right|^{2} .
\end{gathered}
$$

By Lemma 2.5, this implies

$$
\lim _{\delta \rightarrow 0} \delta^{-1}\left(\lim _{\rho \rightarrow 0} S_{0}^{(k)}(\epsilon, L ; \rho)-d \pi^{-2} \delta B_{d} U(\epsilon+\delta k)(\epsilon+\delta k)^{d / 2-1}\right)=0 .
$$

Therefore

$$
\lim _{\rho \rightarrow 0} S_{0}(\epsilon, L ; \rho)=\lim _{\delta \rightarrow 0} \sum_{k=0}^{A-1} V(\epsilon+\delta k) \delta
$$

where

$$
V(t)=d \pi^{-2} B_{d} U(t) t^{d / 2-1}=d \pi^{-2} B_{d} t^{-3 / 2} \sin ^{2}(\pi \sqrt{t}), \quad t>0 .
$$

Since $V$ is continuous on $[\epsilon, L]$, the limit on the right-hand side of (33) equals $\int_{\epsilon}^{L} V(t) d t$, as claimed.

Proof of Lemma 3.2. By virtue of (30) and (31), it follows from Lemma 3.4 that

$$
\lim _{\rho \rightarrow 0} D(\rho, M)=d \pi^{-2} B_{d} \int_{0}^{\infty} \frac{\sin ^{2}(\pi \sqrt{t})}{t^{3 / 2}} d t=d B_{d},
$$

as required. 
3.2.2. Proof of Theorem 1.4. Let $M=T^{d}$ and let $\rho \gg T^{-\sigma}$, for some $\sigma \in(0,1)$, so that $M \rho \rightarrow \infty$ as $\rho \rightarrow 0$. Lemmas 3.2 and 3.3 imply that $\left\langle|S(\cdot, \rho ; M)|^{2}\right\rangle_{T} \rightarrow d B_{d}$ as $\rho \rightarrow 0$. Then one concludes the proof by using Lemma 3.1 .

Acknowledgements. The first author was supported by a research fellowship from University of Birmingham and the second author was supported by EPSRC grant EP/D00022X/2.

\section{References}

1. P. Bleher and J. Bourgain, Distribution of the error term for the number of lattice points inside a shifted ball. In Analytic Number Theory, Vol. 1 (Allerton Park, IL, 1995) (Progress in Mathematics 3), Birkhäuser (Boston, 1996), 141-153.

2. P. M. Bleher, Z. Cheng, F. J. Dyson and J. L. Lebowitz, Distribution of the error term for the number of lattice points inside a shifted circle. Comm. Math. Phys. 154(3) (1993), 433-469.

3. P. Bleher and F. Dyson, Mean square limit for lattice points in a sphere. Acta Math. 67(3) (1994), 461-481.

4. P. Bleher and F. Dyson, Mean square value of exponential sums related to representation of integers as sum of two squares. Acta Math. 68(3) (1994), 71-84.

5. P. Bleher and J. L. Lebowitz, Variance of number of lattice points in random narrow elliptic strip. Ann. Inst. H. Poincaré Probab. Statist. 31 (1995), 27-58.

6. D. R. Heath-Brown, The distribution and moments of the error term in Dirichlet divisor problem. Acta Arith. 60 (1992), 389-415.

7. C. P. Hughes and Z. Rudnick, On the distribution of lattice points in thin annuli. Int. Math. Res. Not. 13 (2004), 637-658.

8. V. Jarnik, Über die Mittelwertsätze der Gitterpunktlehre. V. Časopis Pěst. Mat. Fys. 69 (1940), 148-174.

9. J. Marklof, The Berry-Tabor conjecture. In Proceedings of the 3rd European Congress of Mathematics (Barcelona, 2000) (Progress in Mathematics 202), Birkhäuser (Basel, 2001), 421-427.

10. J. Marklof, Pair correlation densities of inhomogeneous quadratic forms, II. Duke Math. J. 115 (2002), 409-434.

11. J. Marklof, Pair correlation densities of inhomogeneous quadratic forms. Ann. of Math. (2) 158 (2003), 419-471.

12. J. Marklof, Mean square value of exponential sums related to the representation of integers as sums of squares. Acta Arith. 117 (2005), 353-370.

13. W. M. Schmidt, Approximation to algebraic numbers. Enseign. Math. (2) 17 (1971), 187-253.

14. I. Wigman, The distribution of lattice points in elliptic annuli. Q. J. Math. 57 (2006), 395-423.

Hyunsuk Kang,

Department of Mathematics,

University College London,

Gower Street, London, WC1E 6BT,

U.K.

E-mail: kang.maths@gmail.com
Alexander V. Sobolev,

Department of Mathematics,

University College London,

Gower Street, London, WC1E 6BT,

U.K.

E-mail: avsobolev@math.ucl.ac.uk 